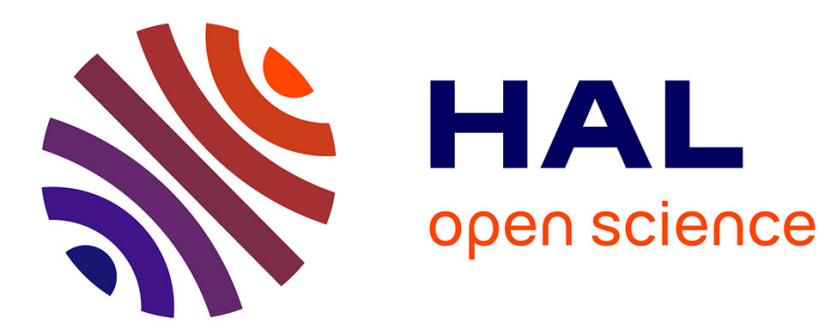

\title{
Les migrations étudiantes dans le système universitaire français au début des années 90 \\ Myriam Baron
}

\section{To cite this version:}

Myriam Baron. Les migrations étudiantes dans le système universitaire français au début des années 90. Revue d'économie régionale et urbaine, 2005, 2, pp.281-300. halshs-00109686

\section{HAL Id: halshs-00109686 \\ https://shs.hal.science/halshs-00109686}

Submitted on 25 Oct 2006

HAL is a multi-disciplinary open access archive for the deposit and dissemination of scientific research documents, whether they are published or not. The documents may come from teaching and research institutions in France or abroad, or from public or private research centers.
L'archive ouverte pluridisciplinaire $\mathbf{H A L}$, est destinée au dépôt et à la diffusion de documents scientifiques de niveau recherche, publiés ou non, émanant des établissements d'enseignement et de recherche français ou étrangers, des laboratoires publics ou privés. 


\author{
LES MIGRATIONS ETUDIANTES DANS LE SYSTEME \\ UNIVERSITAIRE FRANÇAIS AU DEBUT DES ANNEES 90 ${ }^{1}$
}

\title{
STUDENTS' MIGRATIONS IN FRENCH UNIVERSITY SYSTEM IN THE EARLY 90'S
}

\author{
Par \\ Myriam BARON \\ Maître de conférences en géographie \\ UMR CNRS 8504 « Géographie-cités » \\ Université Paris 7 - Denis Diderot \\ UFR GHSS \\ Case courrier 7001 \\ 2 place Jussieu \\ 75251 PARIS CEDEX 05 \\ baron@parisgeo.cnrs.fr
}

Mots clés : système universitaire français, migrations, hiérarchie urbaine

Key words: University system, France, migrations, Urban Hierarchy

Classification JEL : I2, I28, J60, J61, O15, O18, R12, R58

\footnotetext{
${ }^{1}$ Les résultats présentés dans cette note sont issus pour l'essentiel de deux recherches menées à la demande du Ministère de la Recherche, de la Datar et de la Conférence des Présidents d'Université (cf. bibliographie).
} 


\section{INTRODUCTION}

Le début des années 90 a constitué une période charnière, pour ne pas dire majeure, dans le développement et le fonctionnement de l'enseignement supérieur, en particulier pour sa principale composante qu'est l'Université. Cette dernière assurait et continue d'assurer l'accueil d'environ $2 / 3$ des étudiants poursuivant des études supérieures. Et ce même si sa part n'a cessé de baisser au cours de la dernière décennie : on est en effet passé de quelques 1183000 étudiants inscrits à l'Université représentant $69 \%$ de l'ensemble des étudiants de l'enseignement supérieur en 1990-1991 à 1404015 représentant environ 65\% des étudiants inscrits dans l'enseignement supérieur (DEP, 2002). Toutefois, cette baisse de la part relative de l'Université dans le dispositif français de formations supérieures ne doit pas faire oublier que les effectifs y ont crû de près de $19 \%$ au cours de la dernière décennie. Encore convient-il de différencier ce qui s'est passé durant la première moitié des années 90 , durant laquelle la croissance des effectifs a dépassé $25 \%$, alors que durant les 5 années suivantes on a assisté à une baisse de plus de $5 \%$.

C'est dans ce contexte que la mise en place et la montée en puissance du schéma «U 2000 » ont conduit à dresser états des lieux et autres bilans visant à cerner les forces mais aussi les faiblesses du système universitaire français. Celles-ci ont pu être lues au travers d'études qui visaient à caractériser l'offre de formation des différents sites concernés par ce type d'équipement (De Gaudemard (dir.), 1992 ; Brocard et alii (dir.), 1996 ; DATAR (dir.), 1998). Etaient alors différenciés les sites universitaires de première importance qui étaient en mesure d'offrir une grande variété de formations selon les cycles, les disciplines mais aussi les orientations professionnelles ou non, des sites qui n'étaient en mesure d'assurer que des formations correspondant au début du parcours universitaire, voire les seuls premiers cycles à finalités professionnelles (Frémont et alii (dir.), 1992 ; Ertul (dir.), 2000). Or, les forces et les faiblesses du système universitaire français peuvent également être lues au travers des migrations étudiantes qui se produisent entre les différents sites universitaires. Et ce dans la mesure où elles constituent un indicateur pertinent de la connaissance et de l'utilisation du système universitaire par les étudiants. Pour mener des études à partir d'un tel indicateur, encore fallait-il disposer de données collectées à un niveau suffisamment fin : ce ne fut chose faite qu'à la fin des années 90, grâce aux exploitations des données centralisées par la Direction de l'Evaluation et de la Prospective (DEP). Jusqu'à cette date en effet, seules étaient disponibles des données présentées aux niveaux de l'académie ou de la région. Or, en dix ans, la formidable croissance du nombre de sites proposant au moins une formation universitaire a rendu en partie caduque car trop grossière cette seule approche: on est en effet passé d'environ 80 villes abritant au moins une formation universitaire à près de 150. Encore fallaitil définir clairement ce que l'on entendait par mobilité géographique étudiante, ce faisant ce que l'on voulait exactement étudier. Encore fallait-il esquisser quelques pistes possibles de réflexion à partir des principaux résultats de telles études, en particulier les possibilités mises en évidence de sortir du modèle universitaire français réduit pour certains, caricaturé pour d'autres au principe du « Tout partout».

\section{LES MIGRATIONS ETUDIANTES : DEFINITIONS ET ENJEUX}

\section{Cadre et limites de l'étude}

Pourquoi à la fin des années 90 travailler sur des informations collectées au début de la décennie ? Pourquoi ne pas avoir tenté une mise en relation des situations enregistrées tout au long de la période, voire un suivi longitudinal des étudiants inscrits à l'Université ? Parce que 
la décennie 90 a été marquée non seulement par de grands bouleversements du système universitaire français mais aussi par de profonds changements dans l'enregistrement et la collecte des informations concernant les étudiants qui y sont inscrits. Au milieu des années 90, il s'est produit un changement de format dans le traitement de l'information individuelle : on est entre autres passé de la norme EFU valable jusqu'en 1994 à la norme SISE à partir de l'année universitaire 1994-1995: ce qui a rendu le suivi de l'information beaucoup plus difficile notamment pour ce qui concerne le codage des diplômes. De plus, au cours de ces mêmes années, le code permettant d'identifier un étudiant a changé : on est ainsi passé du code INSEE au numéro dit INE (Identifiant National Etudiant). C'est pourquoi il ne paraissait pas raisonnable de mener une étude sur les migrations des étudiants au cours de la période 1995-1998 puisque les changements majeurs dans l'enregistrement des informations risquaient de compromettre le suivi d'un étudiant inscrit à l'Université, suite à. Il faut donc considérer ce premier travail comme un défrichement, un premier cadrage visant à donner quelques ordres de grandeur sur le phénomène et surtout à confirmer ou non quelques hypothèses.

Les informations centralisées par la DEP regroupent en effet ni plus ni moins que les fiches de renseignements pour chaque étudiant inscrit à l'Université. Cette base de données renferme plusieurs informations à caractères géographiques : pour un étudiant sont ainsi recensés le lieu de résidence de ses parents, l'établissement dans lequel il a préparé et obtenu le baccalauréat et bien évidemment la composante de l'université dans laquelle il est inscrit. A partir de ces renseignements géo référencés, on est en mesure de caractériser les aires d'influences et les zones de concurrences des universités. Par exemple, à partir du lieu de résidence des parents, on peut définir, au moins pour les premières années d'étude dans la mesure où la décohabitation entre étudiants et parents n'est pas encore forte, les aires de recrutement des universités. D'autre part, pour les bacheliers de l'année et les nouveaux inscrits à l'université, on peut cerner les migrations associées à la poursuite d'études supérieures.

\section{La migration des étudiants : une définition parmi d'autres}

L'exemple des bacheliers de l'année souligne l'importance de disposer d'informations à deux dates successives pour pouvoir évaluer une migration durant le parcours universitaire. Et ce a fortiori si l'on envisage les migrations des étudiants comme la traduction des complémentarités et des concurrences entre établissements universitaires. Force est alors de ne prendre en considération que les étudiants présents dans les universités durant deux années successives. On ne raisonne plus sur les quelques 1413600 étudiants inscrits dans les universités en 1993 mais sur les 976055 qui ont poursuivi leurs études au sein du système universitaire métropolitain au cours de l'année 1994-1995. Pour ce dernier ensemble, on est en mesure d'indiquer s'il y a eu ou non mobilité géographique à partir de la comparaison des lieux d'étude pour les deux années consécutives. Encore faut-il s'entendre sur ce que l'on définit comme mobilité géographique. C'est pourquoi, tout au long de ce travail, un étudiant inscrit à l'Université a été considéré comme migrant quand le changement de lieu d'étude a correspondu au passage d'une ville universitaire à une autre ville universitaire. La ville en question étant définie au sens de l'unité urbaine de l'INSEE, à savoir la ville centre et les communes de l'agglomération. Un changement d'université peut donc ne pas être enregistré comme une migration dans le cas où les deux établissements sont localisés dans la même ville. Par exemple, deux inscriptions successives à l'Université Paris 6 - Pierre et Marie Curie puis, l'année suivante, à l'Université Paris 7 - Denis Diderot, toutes deux localisées dans l'agglomération parisienne, ne sont pas enregistrées comme une migration. A l'inverse, la migration peut ne pas s'accompagner d'une inscription dans un autre établissement, quand 
une même université offre des lieux d'étude sur plusieurs villes : la migration d'un étudiant de Bayonne à Pau est bien enregistrée comme une migration, sans qu'il y ait pour autant inscription dans une université différente puisque les deux centres dépendent de l'Université de Pau et des Pays de l'Adour. Cette définition de la migration ne prend en compte que le changement de lieu d'étude : elle ne dit rien du changement de résidence des étudiants, qui demeure inconnu.

\section{Un phénomène très limité}

Pour les deux années universitaires retenues, l'étude a ainsi porté sur quelques 976055 étudiants par rapport auxquels ont été définis les déplacements de 58500 étudiants migrants, soit $6 \%$ de l'ensemble de ceux qui poursuivent des études. Les migrations des étudiants entre pôles universitaires semblent n'être qu'un phénomène restreint voire marginal ne concernant que 6 étudiants sur 100. On pourra certes souligner que ce faible taux de mobilité peut être mis en regard avec le fait que le territoire de l'étude n'inclut qu'un petit nombre d'antennes universitaires identifiées comme des pôles autonomes (cf. Figure $\mathrm{n}^{\circ} 1$ ). En France métropolitaine en effet, sur les 124 sites abritant au moins une unité de formation universitaire lors d'une des deux années d'étude, 51 n'apparaissaient qu'en 1994-1995 : rien pour l'année précédente. Certes, cette période a été marquée par de nombreuses ouvertures de sites universitaires, mais, dans le même temps, on notait de grandes réticences de la part des établissements pour enregistrer les inscriptions aux lieux des antennes et non au siège des universités mères. Au final, n'ont pu être conservées pour l'étude que les 73 agglomérations métropolitaines communes aux deux années d'étude, soit un peu moins de $60 \%$ des pôles universitaires présents à l'une des deux dates.

Tout ceci laisse à penser que les flux d'étudiants au passage de l'année universitaire 19931994 à l'année 1994-1995 ont été sous-estimés. Mais dans quelles proportions ? Difficile d'y répondre. Et ce même si les premières tentatives d'actualisation de ce travail indiquent que, pour les années 2001-2002 et 2002-2003, la part des migrants dans l'ensemble des étudiants poursuivant des études à l'université n'atteignait pas $8 \%$ : tout ceci invite à reconsidérer le statut de la mobilité dans le parcours de formation universitaire. Pendant longtemps en effet, on a considéré que le fait de migrer, de voir du pays faisait partie du cursus universitaire dans la mesure où cela correspondait à un enrichissement de la formation reçue. Si un tel argumentaire doit être maintenu, il ne concerne qu'une très petite fraction de la population étudiante.

Plus avant, quelques cadrages de ces migrations étudiantes viennent à l'encontre de quelques idées reçues. $75 \%$ des migrations se font entre agglomérations de province et $25 \%$ entre Paris et la province. Dans ce dernier cas, les échanges s'équilibrent avec un léger bénéfice $(500$ étudiants) au profit de l'agglomération parisienne. La ponction de l'agglomération parisienne sur les étudiants de province n'est pas aussi importante qu'on pouvait le penser, en particulier au moment du passage en troisième cycle. Près de $14 \%$ des étudiants parisiens de $2^{\text {ème }}$ cycle qui poursuivent leurs études en $3^{\text {ème }}$ cycle migrent vers la province, $21 \%$ des étudiants de province rejoignent une autre ville de province, tandis que $6 \%$ d'entre eux se dirigent vers Paris. Un tel constat conduit à réinvestir la question relative à l'équilibre des relations entre Paris et la province. Pendant longtemps en effet, la problématique des migrations étudiantes s'est largement focalisée sur les rapports Paris-province, s'articulant ainsi sur une question récurrente de l'aménagement du territoire français. A l'origine d'un débat souvent passionné résidait un modèle de référence qui présentait ces relations comme particulièrement dissymétriques. Le complexe universitaire parisien était censé exercer sur la province une 
ponction puissante et sélective. Cette dernière allant s'accentuant du premier au troisième cycle. Mais, outre que la comparaison des offres de formation n'est pas entreprise aisée, l'absence de toute évaluation des migrations étudiantes a pu laisser le champ libre à des points de vue faiblement étayés s'appuyant sur la connaissance soit d'une sous-population étudiante particulière, soit d'un établissement particulier.

\section{Figures $n^{\circ} 1$ : Les villes universitaires retenues pour l'étude}

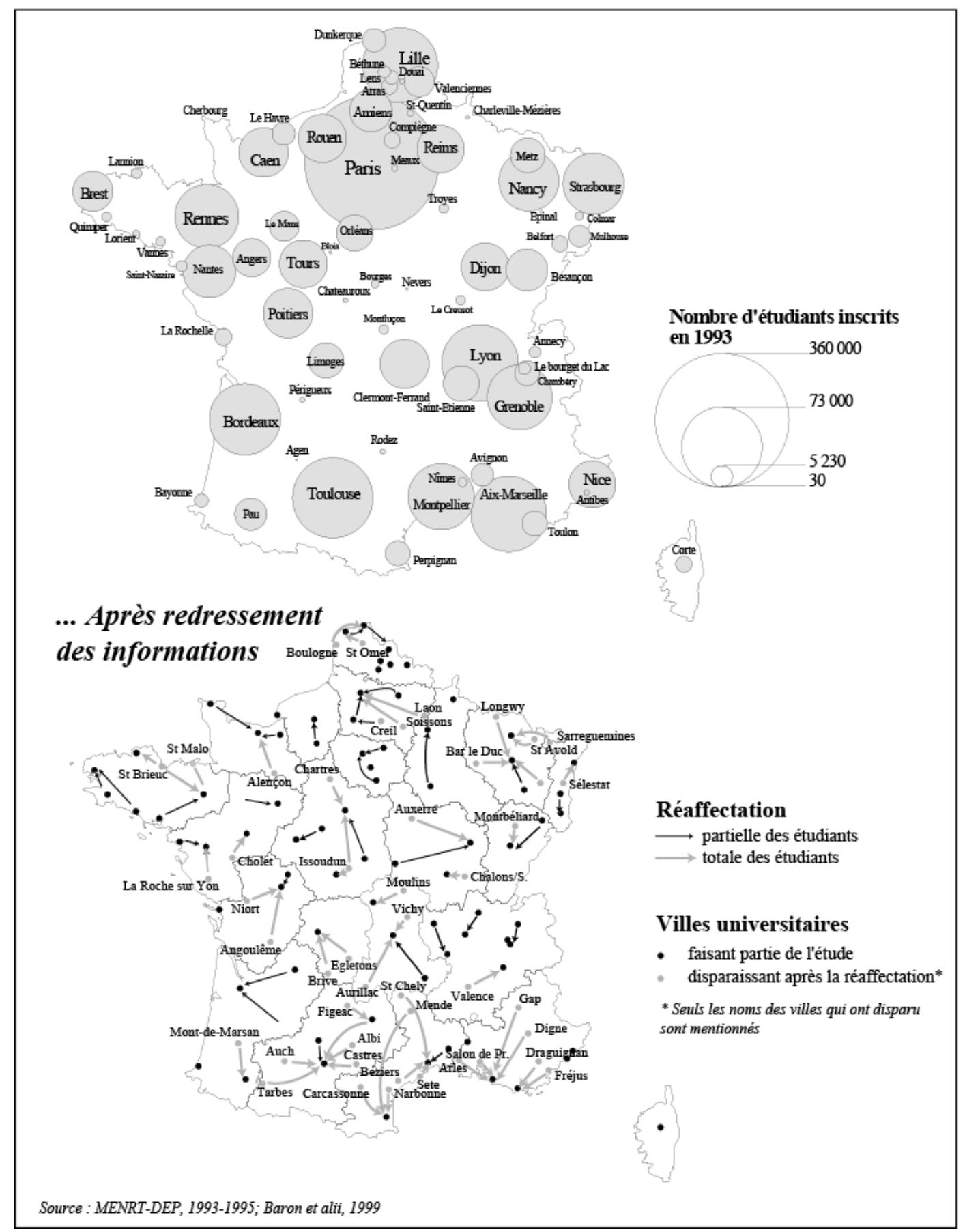




\section{PRINCIPALES CARACTERISTIQUES DES MIGRATIONS ETUDIANTES}

Il n'en demeure pas moins que les flux d'étudiants les plus importants relient les grands pôles universitaires entre eux. Les plus grands d'entre eux apparaissent d'une part entre Paris et la plupart des grandes villes universitaires de province (Grenoble, Lyon, Marseille, Toulouse, Bordeaux, Rennes, Lille, Nancy et Strasbourg), et d'autre part entre ces dernières surtout quand elles relèvent d'un même sous-ensemble régional (structuré autour de quelques grands pôles tels Bordeaux, Montpellier, Marseille, Lyon, Grenoble, Strasbourg, Nancy ou Lille). L'analyse systématique des échanges d'étudiants entre les 73 villes retenues pour l'étude confirme que deux facteurs principaux exercent une influence décisive sur le volume de ces flux (cf. Encadré $\left.n^{\circ} 1\right)$.

\section{Encadré $n^{\circ} 1$ : Hypothèses retenues pour la modélisation des migrations étudiantes}

(a) Le choix de modèles d'interaction à double contrainte

A la différence des modèles d'interaction spatiale de type gravitaire qui se donnent pour seule règle de reconstituer la somme totale des flux de migrants entre les villes françaises, les modèles à double contrainte imposent la conservation de l'ensemble des marges de la matrice d'échanges. Les modèles à double contrainte supposent connues les quantités d'étudiants émises ou reçues par chaque ville. Ils ne s'intéressent donc pas à l'attractivité ou la répulsivité globale des villes mais aux systèmes de relations spécifiques qui s'établissent entre certaines d'entre elles.

\section{(b) modèles agrégés et modèles désagrégés}

Sachant que la population étudiante se compose de sous-populations qui sont à différentes étapes de leur cursus universitaire, on peut choisir soit :

(1) d'établir un modèle moyen de comportement en utilisant un modèle unique qui sera appliqué à l'ensemble des flux étudiants entre les villes (modèle agrégé).

(2) de modéliser séparément les mouvements migratoires des étudiants engagés dans chacun des cycles et reconstituer à partir de là un comportement résultant, qui est la somme des comportements particuliers (modèle désagrégé).

Même si les modèles désagrégés fournissent en général des ajustements de moins bonne qualité que le modèle agrégé (car ils portent sur des effectifs plus réduits), la somme des modèles désagrégés fournit une estimation plus juste des migrations que le modèle agrégé. C'est donc la solution qui a été retenue dans le cadre de ce travail.

\section{(c) Le modèle de référence et ses variantes}

Le modèle migratoire de référence est un modèle d'interaction spatiale à double contrainte (1) ne faisant intervenir que les masses d'émigrants ou d'immigrants et la distance à vol d'oiseau comme facteurs explicatifs :

$(1) F_{i j}=a_{i .} . O_{i} \cdot b_{j} \cdot D_{j} \cdot d_{i j}{ }^{-\alpha}$ modèle d'interaction spatiale avec

$\mathrm{F}_{\mathrm{ij}}$ : flux estimé entre les villes i et $\mathrm{j}$

$\mathrm{O}_{\mathrm{i}}$ : nombre d'étudiants ayant quitté la ville i en 1993-94

$\mathrm{D}_{\mathrm{j}}$ : nombre d'étudiants arrivés dans la ville $\mathrm{j}$ en 1994-95

$\mathrm{d}_{\mathrm{ij}}$ : distance à vol d'oiseau entre les villes $\mathrm{i}$ et $\mathrm{j}$

$a_{i}, b_{j}$ : vecteurs de paramètres assurant la conservation des origines et des destinations

$\alpha$ : paramètre à estimer reflétant le frein imposé par la distance aux déplacements

Deux modèles, l'un plus simple, l'autre plus complexe ont été utilisés pour mieux cerner le rôle de la distance et le rôle de l'appartenance régionale.

Le modèle sans interaction spatiale (2) ne fait intervenir que les capacités d'émission et de réception des villes et permet de mesurer le gain d'explication que procure l'ajout de la distance à vol d'oiseau dans le modèle. 
$(2) F^{*}{ }_{i j}=a_{i .} \cdot O_{i} \cdot b_{j} . D_{j}$ modèle sans interaction spatiale

Le modèle d'interaction spatiale et d'appartenance régionale (3) ajoute à l'effet de la distance celui de l'appartenance régionale commune, et permet d'évaluer la valeur moyenne de l'effet de barrière $\gamma$ qui est subi par les migrants lorsqu'ils passent d'une région à une autre. Un effet de barrière $\gamma=2$ signifie par exemple que, à distance et masses égales, les flux inter-régionaux sont en moyenne deux fois plus faibles que les flux intra régionaux.

$(3) F_{i j}^{*}=a_{i} \cdot O_{i} \cdot b_{j} \cdot D_{j} \cdot d_{i j}{ }^{-\alpha} \cdot \gamma^{A i j}$ modèle d'interaction spatiale et d'appartenance régionale avec $\mathrm{A}_{\mathrm{ij}}=1$ si les villes $\mathrm{i}$ et $\mathrm{j}$ sont localisées dans la même région et $\mathrm{Aij}=0$ dans le cas contraire

Tous ces modèles ont été estimés par régression poissonienne selon la méthode du maximum de vraisemblance. Cette méthode d'ajustement est beaucoup plus robuste que les méthodes habituelles d'estimation fondées sur des régressions log-linéaires et elle permet de prendre en compte les flux nuls.

\section{Le rôle déterminant ...}

\section{1....de l'importance des pôles universitaires}

En premier lieu, l'effet des masses démographiques concernées, c'est à dire la capacité d'émission de la ville de départ et la capacité de réception de la ville d'arrivée, constitue le déterminant le plus évident de la migration dans une logique de régulation (cf. Tableau $\mathrm{n}^{\circ} 1$ ). Autrement dit, le simple jeu de l'offre et de la demande de formation explique à lui seul en moyenne $68 \%$ des migrations étudiantes. Toutefois, il convient de souligner que l'offre et la demande de formation jouent un rôle d'autant plus faible que les étudiants changent de ville universitaire au niveau du premier cycle : elles ne permettent de décrire que $43 \%$ des migrations étudiantes. A l'opposé, pour les fins de parcours universitaires, ces mêmes offres et demandes de formation expliquent à elles-seules environ $80 \%$ des migrations étudiantes. Les masses démographiques des villes universitaires voient donc leur importance presque doubler dans la description des migrations étudiantes entre le début et la fin du cursus universitaire.

Tableau $n^{\circ} 1$ : Caractéristiques des migrations interurbaines d'étudiants

\begin{tabular}{|c|c|c|c|c|c|c|c|c|}
\hline \multirow{3}{*}{$\begin{array}{l}\text { Type de } \\
\text { modèle } \\
\text { d'interactio } \\
\text { n spatiale }\end{array}$} & \multicolumn{2}{|c|}{$\begin{array}{l}\text { Parcours d'étude } \\
\text { ou passage }\end{array}$} & \multicolumn{2}{|c|}{$\begin{array}{l}\text { Nombre d'unités } \\
\text { urbaines }\end{array}$} & \multicolumn{4}{|c|}{ Modèles avec évaluation des flux en fonction } \\
\hline & \multirow[t]{2}{*}{ De 1993} & \multirow[t]{2}{*}{ A 1994} & \multirow[t]{2}{*}{$\begin{array}{l}\text { Au } \\
\text { départ }\end{array}$} & \multirow[t]{2}{*}{$\begin{array}{l}\text { A } \\
\text { l'arrivée }\end{array}$} & \multirow{2}{*}{$\begin{array}{l}\begin{array}{l}\text { Des masses } \\
\text { d'émigrants et } \\
\text { d'immigrants }\end{array} \\
\begin{array}{c}\text { Qualité de } \\
\text { l'ajustement } \mathrm{R}^{2} \\
(\%)\end{array}\end{array}$} & \multicolumn{3}{|c|}{$\begin{array}{l}\text { Des masses d'émigrants, d'immigrants, } \\
\text { de la distance et de l'appartenance } \\
\text { régionale }\end{array}$} \\
\hline & & & & & & $\begin{array}{l}\text { Qualité de } \\
\text { l'ajustement } \\
\mathrm{R}^{2}(\%)\end{array}$ & $\begin{array}{l}\text { Frein de } \\
\text { la distance } \\
(\alpha)\end{array}$ & $\begin{array}{l}\text { Effet } \\
\text { d'appartenance } \\
\text { régionale }(\gamma)\end{array}$ \\
\hline \multirow[t]{6}{*}{ Désagrégé } & $\mathbf{1}^{\mathrm{er}}$ cycle & $1^{\mathrm{er}}$ cycle & 69 & 72 & 43 & 86 & $-1,48$ & 3,78 \\
\hline & $1^{\text {er }}$ cycle & $2^{\mathrm{e}}$ cycle & 68 & 59 & 55 & 84 & $-1,15$ & 2,87 \\
\hline & $2^{\mathrm{e}}$ cycle & $2^{\mathrm{e}}$ cycle & 55 & 58 & 64 & 83 & $-1,07$ & 2,94 \\
\hline & $2^{\mathrm{e}}$ cycle & $3^{\mathrm{e}}$ cycle & 52 & 47 & 79 & 91 & $-0,95$ & 1,80 \\
\hline & $3^{\mathrm{e}}$ cycle & $3^{\mathrm{e}}$ cycle & 46 & 49 & 81 & 91 & $-0,99$ & 2,39 \\
\hline & \multicolumn{2}{|c|}{ Autres } & 58 & 60 & 67 & 82 & $-1,26$ & 3,52 \\
\hline Agrégé & & & 73 & 74 & 68 & 91 & $-1,14$ & 3,18 \\
\hline
\end{tabular}




\section{2....de la distance qui les sépare et de leur appartenance à une même région}

Ensuite, l'effet de la proximité spatiale, c'est à dire la décroissance des probabilités de relation entre les étudiants de deux villes en fonction de la distance qui les sépare, constitue sans aucun doute le second déterminant de ces mêmes migrations. A l'instar du reste de la population française, les étudiants tendent en moyenne à privilégier les destinations proches par rapport aux destinations lointaines. L'effet dissuasif joué par la distance diminue de moitié et régulièrement du premier au troisième cycle, passant de $-1,48$ à $-0,95$. Cette décroissance des probabilités de migration avec la distance est liée à des déterminants économiques ou géographiques objectifs (coûts de déplacements, information plus importante sur les destinations proches que sur les destinations éloignées etc.) ainsi qu'à des facteurs psychologiques plus difficilement mesurables (attachement au lieu d'origine, volonté de maintenir des liens antérieurs avec des parents ou des amis etc.) et à des héritages (migrations antérieures de populations étudiantes ou non susceptibles d'accueillir le migrant).

Enfin, bien que leurs influences soient sans commune mesure avec celle des deux précédents, d'autres facteurs de nature plus qualitative peuvent être introduits pour décrire plus exactement les migrations des étudiants entre les villes de province. En particulier l'effet de "proximité territoriale », c'est à dire le fait qu'à distance égale les migrants ont tendance à rester à l'intérieur des limites de leur entité régionale ou académique d'appartenance est une réalité tangible (cf. Tableau $\mathrm{n}^{\circ} 1$ ). Toutes choses égales quant à la distance et à l'offre de formation, l'ensemble des étudiants migrants a trois fois plus de chance de choisir une destination localisée à l'intérieur de leur propre région plutôt que dans une autre région (effet d'appartenance régionale égal à $+3,18$ ). Comme le frein aux déplacements joué par la distance, l'effet d'appartenance régionale dépend fortement du niveau atteint dans le parcours universitaire : il décroît ainsi de moitié du premier cycle $(+3,78)$ au passage entre deuxième et troisième cycle $(+1,80)$. Au total, l'effet conjugué de la taille des pôles universitaires, de la distance qui les sépare et de leur appartenance à une même région explique $91 \%$ des migrations étudiantes (cf. Tableau $\mathrm{n}^{\circ} 1$ ).

\section{Les migrations non banales: les spécificités géographiques des déplacements des étudiants}

Dans chacun des cas, on peut en outre calculer et interpréter les écarts qui apparaissent entre ces migrations « banales », dans la mesure où elles ne dépendent que de l'importance des pôles universitaires, de la distance qui les sépare et de leur appartenance régionale, et les migrations effectivement enregistrées. Ces écarts sont l'expression de dimensions plus spécifiques des migrations. Ils révèlent l'existence de relations interurbaines non banales qui, selon les directions, tirent leur force de segments particuliers de la migration, en rapport avec les différentes étapes du parcours d'étude (cf. Encadré $\mathrm{n}^{\circ} 2$ ). Dans un contexte de relations inter-universitaires de plus en plus concurrentielles mais aussi complémentaires, ces parts spécifiques des migrations interurbaines pourraient être interprétées comme la marge de liberté dont disposent les institutions pour infléchir le fonctionnement d'ensemble du système migratoire.

\section{Encadré $n^{\circ} 2$ : Méthode de caractérisation des migrations résiduelles}

Ayant ajusté autant de modèles d'interaction spatiale à double contrainte qu'il y avait de souspopulations définies par la position dans les cycles d'étude $(11,12,22,23,33$ et autres), on se trouve en présence de six matrices de flux observés (notées $\left.\mathrm{F}^{1} . . \mathrm{F}^{6}\right)$ et de six matrices de flux estimées $\left(\mathrm{E}^{1} . . \mathrm{E}^{6}\right)$ qui définissent par soustraction six matrices de flux résiduels (notées $R^{1} . . R^{6}$ ). L'intérêt de cette 
décomposition est de permettre une évaluation à la fois quantitative et qualitative des migrations résiduelles entre les villes qui débouche sur une typologie des comportements migratoires non banaux.

Une analyse en composantes principales effectuée sur le tableau des paires de villes décrites par les résidus aux différentes étapes du cycle universitaire a permis de dégager deux dimensions principales :

* Le premier facteur, de loin le plus important, exprime l'existence de sur-migrations ou de sous-migrations entre les villes, tous cycles confondus. Ce facteur est étroitement corrélé avec le résidu migratoire total $\mathrm{R}^{\text {tot }}$, c'est-à-dire la somme des résidus affectant l'ensemble des sous-population étudiantes. Les coordonnées sur ce premier facteur permettent de définir deux situations de migrations non banales (A et B) et une situation de migration banale (C)

(A) la somme totale des migrations d'étudiants observées entre deux villes sur l'ensemble des cycles est significativement supérieure à ce que prévoit le modèle

(B) la somme totale des migrations d'étudiants observées entre deux villes sur l'ensemble des cycles est significativement inférieure à ce que prévoit le modèle

(C) la somme totale des migrations d'étudiants observées entre deux villes sur l'ensemble des cycles est globalement conforme à ce que prévoit le modèle (même si cette conformité globale peut résulter de compensations entre des excédents sur certains cycles et des déficits sur d'autres).

* Le second facteur, de nature plus qualitative, permet de préciser l'influence du niveau d'étude sur la constitution des excédents ou des déficits migratoires entre les villes. Il oppose en effet les cycles d'étude 1.1 et 1.2 aux cycles d'étude 2.3 et 3.3. Croisé avec le critère précédent, il permet de définir six types de relations migratoires non-banales entre les villes.

A.1 : sur-migration concernant principalement les premiers cycles

A. 2 : sur-migration concernant l'ensemble des cycles

A. 3 : sur-migration concernant principalement les cycles supérieurs

B. 1 : sous-migration concernant principalement les premiers cycles

B. 2 : sous-migration concernant l'ensemble des cycles

B. 3 : sous-migration concernant principalement les cycles supérieurs

Pour ne pas compliquer l'analyse, nous avons choisi de ne pas cartographier ou étudier les situations de type $\mathrm{C} 1$ ou $\mathrm{C} 3$ qui correspondent pourtant à des situations non banales (présence simultanée de résidus positifs sur certains cycles et négatifs sur d'autres).

N.B. : comme toute classification, la méthode proposée ci-dessus présente un certain degré d'arbitraire et une très légère modification des seuils retenus pourrait faire passer un flux entre deux villes d'une catégorie à une autre. Il faut donc s'attacher davantage aux configurations d'ensemble qui apparaissent qu'aux flux particuliers observables entre tels ou tels couples de villes

\subsection{Sous-migrations et barrières géographiques}

C'est ainsi que la forte sous-migration étudiante au départ ou à destination de Paris, dans le Bassin parisien d'une part et dans le Nord d'autre part, retient l'attention (cf. Figures $\mathrm{n}^{\circ} 2$ ). Exceptionnellement élevées, les capacités de rétention réciproque de Paris et de chacun des centres de ces régions peuvent difficilement être expliquées par les potentiels locaux d'offre de formation. Il faut sans doute faire intervenir des spécificités régionales qui dépassent largement le champ des migrations étudiantes. Le frein à ces migrations ne se retrouve-t-il pas dans les migrations générales de la population? ( Pumain et Saint-Julien, 1989). Les migrations étudiantes ne feraient alors que retrouver les traces de pratiques migratoires régionales beaucoup plus générales et assez enracinées sur la durée, puisqu'on peut en repérer la spécificité depuis un demi-siècle au travers des études sur les migrations interurbaines de population. Pas plus que le Bassin parisien et le Nord ne sont terres d'immigration pour les Parisiens, Paris ne représente une direction préférentielle pour les émigrants qui quittent une ville des régions de la périphérie de l'Ile-de-France. 
Figures $n^{\circ} 2$ : Effets de barrières migratoires

a. Entre Paris et les villes universitaires de province

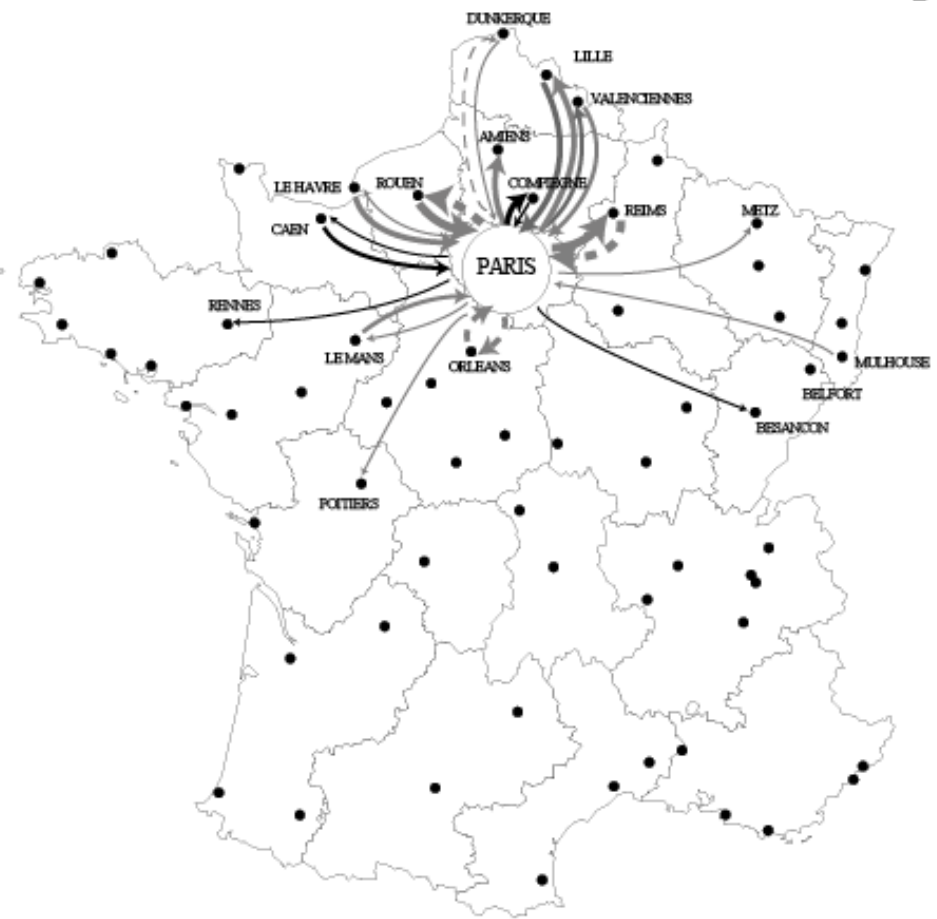

La sous-migration est comprise

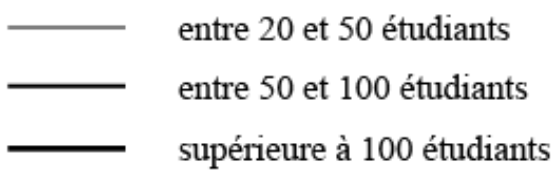

b. Entre villes universitaires de province

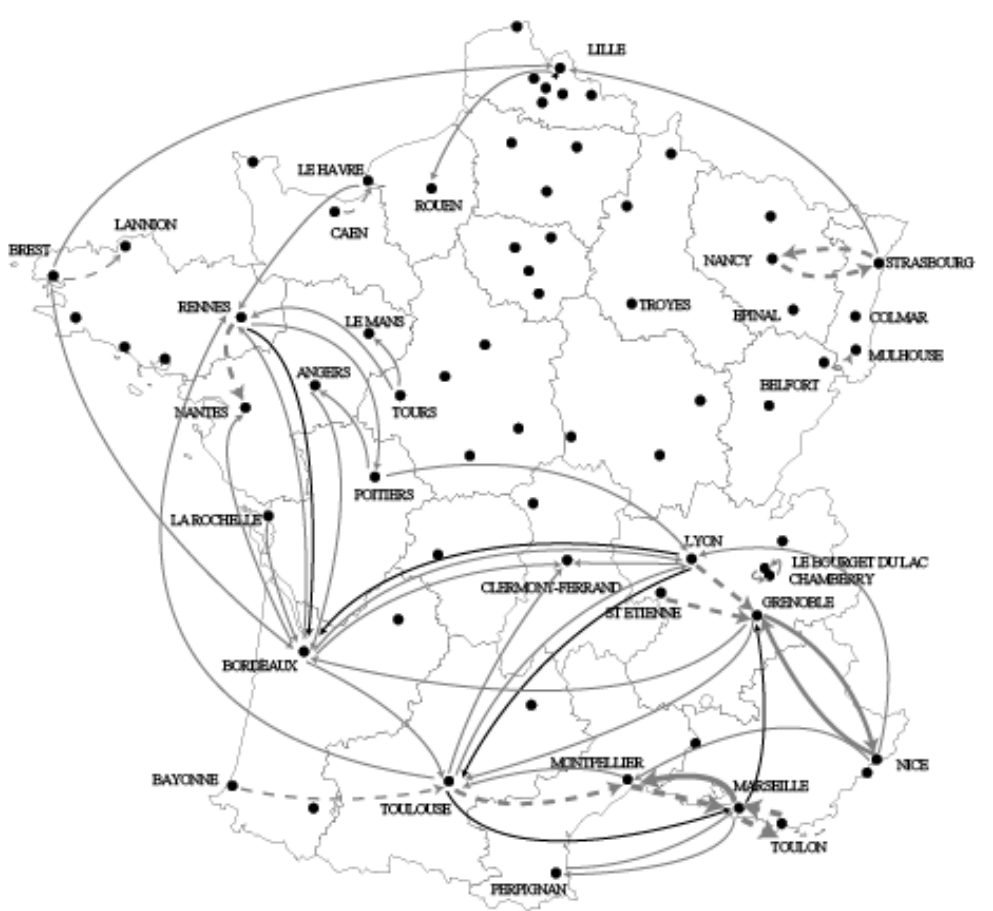

\section{La sous-migration est plutôt imputable}

- - - - aux 1ers cycles

à tous les cycles

$\longrightarrow$ aux $2 \mathrm{e}$ ou $3 \mathrm{e}$ cycles 
Les sous-migrations entre villes de province révèlent quant à elles un jeu de barrières à différents échelons géographiques. Entre villes éloignées, ces barrières soulignent de véritables limites interrégionales. Une barrière importante apparaît au niveau du Massif Central entre grands centres de l'Est et de l'Ouest de la moitié sud du pays. Des freins parfois importants apparaissent aussi dans les migrations entre couples de villes « voisines » et donc aussi plus directement concurrentes. Ces barrières peuvent être inscrites dans la longue durée des histoires urbaines et fortement intégrées dans les représentations que chacun a des distances dans le voisinage. Les caractéristiques qualitatives de ces migrations non réalisées sont à considérer dans la mesure où, pour la majorité des directions concernées, tous les cycles subissent un même effet de barrière.

\subsection{Sur-migrations et directions préférentielles}

De la même manière, les directions préférentielles des sur-migrations sont relativement aisées à repérer. Elles concernent tout d'abord les échanges entre Paris et les grandes villes universitaires de province les plus éloignées, méridionales en particulier. Ce schéma rappelle à nouveau celui bien connu des migrations générales de population : ce qui ne peut qu'inciter au rapprochement des deux structures de migrations.

Entre villes de province, les flux préférentiels ont tendance à dessiner des sortes de systèmes régionaux de préférences. Ces derniers peuvent varier, soit en ne reliant que deux centres comme Perpignan et Montpellier, soit en révélant des formes de polarisation régionale plus nette autour d'un centre comme dans le cas de Rennes. Les systèmes de préférences géographiques organisés autour de Lille, Lyon, Bordeaux ou Marseille sont un peu plus complexes à la fois par le nombre de centres reliés au pôle principal, et par la formation de systèmes régionaux plus "réticulés». Au total, ces systèmes assez fortement intégrés peuvent avoir une large extension territoriale.

Le schéma des migrations étudiantes entre agglomérations ressemble fortement à celui que l'on observe pour les migrations de l'ensemble de la population sur le territoire français. En d'autres termes, ce schéma se définit largement à partir du jeu des facteurs liés à l'offre/demande des pôles universitaires et à la distance qui les sépare. Sachant que les centres universitaires de petite taille sont en général ceux qui disposent de l'offre de formation la moins diversifiée, ces résultats conduisent à se demander dans quelle mesure les migrations étudiantes constituent un vecteur des ajustements géographiques entre offre et demande de formation universitaire.

\section{LES MIGRATIONS ETUDIANTES "CONTRAINTES": AMPLEUR ET SIGNIFICATIONS}

A ce titre, la trajectoire d'étude associée à la migration des diplômés des I.U.T. interpelle par ses modalités particulières. Ces migrants représentent d'une part plus de $30 \%$ des titulaires d'un diplôme de premier cycle s'inscrivant en deuxième cycle et d'autre part près de $45 \%$ des titulaires d'un D.U.T. qui poursuivent des études en deuxième cycle. Ainsi, pour un titulaire de D.U.T. sur deux, la poursuite d'étude à l'Université correspond à un changement de ville universitaire. Cette migration est largement contrainte. On dira parfois qu'elle est " différée », puisqu'elle résulte d'un rapprochement inévitable des lieux d'offre de formation de deuxième cycle. 


\section{Définir les migrations contraintes}

A partir de cet exemple, il parait essentiel de distinguer dans les migrations étudiantes celles qui relèvent d'un choix préférentiel mettant en concurrence l'offre présente dans le pôle universitaire d'origine de l'étudiant et l'offre présente dans d'autres villes et celles qui relèvent d'une contrainte liée à une offre de formations insuffisante au lieu de départ c'est à dire à une incompatibilité entre le choix de poursuite d'étude effectué par l'étudiant et les formations proposées dans sa ville d'origine (cf. Encadré $\mathrm{n}^{\circ} 3$ ). A l'échelon de chaque cycle d'étude, de chaque discipline ou de chaque type de formation, l'offre est en effet inscrite dans un schéma territorial plus ou moins particulier. Les contraintes à l'émigration apparaissent ainsi d'autant plus grandes que les étudiants se situent sur un segment de la formation dont l'offre est plus inégalement répartie sur le territoire et/ou géographiquement peu intégrée. La contrainte à l'émigration atteint à des degrés variés les différents secteurs auxquels appartiennent les disciplines (cf. Tableau $\mathrm{n}^{\circ} 2$ ). Le secteur des Sciences est le plus concerné. Ce sous-ensemble cumule à la fois les taux d'émigration et les taux de contrainte les plus élevés. Ainsi, alors qu'il ne concentre que $27 \%$ des étudiants non migrants et $33 \%$ des émigrants non contraints, ce sous-ensemble réunit à lui seul plus de la moitié des émigrants contraints $(55 \%)$. Les niveaux élevés de ces taux peuvent dépendre non seulement d'une offre géographiquement segmentée des différentes disciplines appartenant au secteur Sciences, mais aussi des réorientations vers d'autres disciplines extérieures à ce même secteur. A l'inverse, les étudiants inscrits dans le secteur Lettres et Sciences Humaines apparaissent à la fois peu mobiles (taux d'émigration égal à $4 \%$ ) et relativement peu contraints à quitter leur ville universitaire d'origine (émigration contrainte égale à $16,2 \%$ ).

\section{Encadré $n^{\circ} 3$ : Détermination de la part des migrations contraintes par la méthode du choix} révélé

La méthode du choix révélé, que nous avons utilisée pour déterminer les migrations contraintes, se fonde sur l'hypothèse forte que la formation effectivement suivie par un étudiant au terme de sa migration correspond bien à la formation souhaitée par ce dernier. Cette méthode postule donc que le choix de la formation universitaire précède la décision de migrer, c'est-à-dire qu'il peut en constituer la cause mais n'en est généralement pas la conséquence. En d'autres termes, un étudiant qui suivait des études dans la ville $A$ au cours de l'année universitaire 1993-94 et qui migre vers une ville $B$ pour poursuivre ses études en 1994-95 aura effectué une migration contrainte si les études qu'il suit en 1994-95 dans la ville $B$ ne sont pas disponibles cette même année dans sa ville d'origine $A$. On considérera en effet que les études suivies en 1994-95 dans la ville B constituent le choix révélé de formation de l'étudiant et que ce choix est incompatible avec l'offre de formation disponible dans la ville d'origine.

Cette méthode est évidemment imparfaite puisqu'elle privilégie a priori le choix de la formation universitaire dans la décision de migrer, négligeant les autres motivations qui ont pu conduire l'étudiant à modifier son lieu d'étude. Or, il peut arriver qu'un étudiant décide de migrer pour des raisons indépendantes de ses études et soit comptabilisé à tort comme migrant contraint. Ce problème est cependant peu gênant car les migrants comptabilisés à tort comme migrants contraints n'en demeurent pas moins des étudiants qui ont effectué un déplacement vers une ville où la formation qu'ils suivent est incompatible avec celle offerte par leur ville d'origine. Il y a donc simplement dans ce cas coïncidence et non pas causalité entre la migration et la contrainte de formation.

L'étudiant X, inscrit dans la ville A en 1993-94, a migré vers la ville B en 1994-95 pour suivre une formation professionnelle de physique de $2^{e}$ cycle.

Contrainte de niveau 1 (cycle) : Si la ville d'origine du migrant ne possède pas au moins 5 étudiants inscrits en $2^{\mathrm{e}}$ cycle en 1994-95, on considère que l'étudiant X subit une contrainte de premier niveau puisqu'il lui est impossible de trouver une formation correspondant à son cycle d'étude actuel (même en changeant de discipline). 
Contrainte de niveau 2 (cycle*discipline) : Si la ville d'origine du migrant comporte le cycle désiré mais pas la discipline choisie par celui-ci (moins de 5 étudiants en $2^{\mathrm{e}}$ cycle de physique dans la ville A en 1994-95) on considère que l'étudiant subit une contrainte de deuxième niveau, moins forte que la précédente. Il aurait en effet pu éventuellement poursuivre ses études dans sa ville d'origine, mais à condition de changer de discipline.

Contrainte de niveau 3 (cycle*discipline*formation) : Si la ville d'origine du migrant comporte le cycle et la discipline choisie par l'étudiant (au moins 5 étudiants dans une formation professionnelle de $2^{\mathrm{e}}$ cycle de physique) mais pas le type de formation recherchée (moins de 5 étudiants dans une formation professionnelle de $2^{\mathrm{e}}$ cycle de physique), on considère que l'étudiant subit une contrainte de troisième niveau, encore plus faible que la précédente. Il aurait en effet pu poursuivre ses études dans sa discipline, mais à condition d'accepter un type de formation différent (formation générale) de celui qu'il a effectivement choisi au terme de sa migration (formation professionnelle).

Absence de contrainte : Si la ville d'origine du migrant comporte en 1994-95 au moins cinq étudiants dans le cycle, la discipline et la formation qu'il a finalement retenue dans la ville de destination, on considère qu'il a effectué une migration non-contrainte (au sens des trois critères retenus) et qu'il aurait pu poursuivre ses études dans sa ville d'origine. Sa décision de migrer résulte donc soit d'un choix (renommée ou attrait de l'université et de la ville de destination) soit de contraintes non prises en compte par les critères utilisés (sous-discipline de la physique, formation professionnelle spécifique), et pas nécessairement liées au parcours universitaire (logement, rapprochement de conjoints, etc.).

$\mathrm{Au}$ total, sur 58500 étudiants qui ont migré durant leurs études universitaires, ils sont quelques 11500 , soit près de $20 \%$, dont la migration aurait été contrainte du fait de l'inadaptation de l'offre de formation dans la ville de départ. La structure géographique des migrations contraintes ne se réduit pas à un modèle hiérarchisé qui introduirait une dichotomie entre pôles universitaires dominants et pôles dépendants. Ce serait sans compter avec la relative spécialisation des pôles qui se cache derrière chacun, même derrière ceux dont les structures sont apparemment les plus diversifiées. La réalité demeure donc plus complexe.

\section{Tableau $n^{\circ} 2$ : Emigration contrainte et secteurs scientifiques}

\begin{tabular}{|c|c|c|c|c|c|c|}
\hline \multirow[t]{2}{*}{$\begin{array}{c}\text { Secteurs } \\
\text { scientifiques }\end{array}$} & \multicolumn{4}{|c|}{$\begin{array}{c}\text { Dans l'ensemble des émigrants, nombre } \\
\text { d'émigrants contraints }\end{array}$} & \multirow[t]{2}{*}{\begin{tabular}{|c|} 
Total \\
d'émigrants
\end{tabular}} & \multirow{2}{*}{$\begin{array}{c}\text { Taux } \\
\text { d'émigration } \\
(\%)\end{array}$} \\
\hline & $\begin{array}{l}\text { par le } \\
\text { cycle } \\
(\%)\end{array}$ & $\begin{array}{c}\text { par la } \\
\text { discipline } \\
(\%)\end{array}$ & $\begin{array}{c}\text { par le type } \\
\text { de formation } \\
(\%)\end{array}$ & $\begin{array}{c}\text { Total } \\
(\%)\end{array}$ & & \\
\hline $\begin{array}{l}\text { Droit, sciences } \\
\text { économiques }\end{array}$ & 1,0 & 12,1 & 5,1 & 18,3 & 100 & 6,5 \\
\hline $\begin{array}{l}\text { Lettres, sciences } \\
\text { humaines et } \\
\text { sociales }\end{array}$ & 0,1 & 11,1 & 5,0 & 16,2 & 100 & 4,0 \\
\hline Sciences & 1,8 & 17,1 & 11,9 & 30,8 & 100 & 7,9 \\
\hline Santé & 0,0 & 3,5 & 1,4 & 4,9 & 100 & 5,6 \\
\hline Ensemble & 1,0 & 12,9 & 7,3 & 21,1 & 100 & 5,9 \\
\hline
\end{tabular}

Source : Baron et alii, 1998 ; MENRT-DEP, 1993-1995

\section{Complémentarité entre pôles universitaires de tailles comparable...}

On note en premier lieu qu'entre un certain nombre de villes, souvent de grande taille, les flux ne sont pas unidirectionnels (cf. Figure $\mathrm{n}^{\circ} 3$ ). La spécialisation de chacune et les complémentarités, qui en découlent, alimentent des échanges croisés de migrants contraints. 
Sans être toujours symétrique, cette réciprocité, qui se déploie en général entre villes universitaires voisines, est le signe de toute la richesse d'un réseau universitaire qui a su lentement s'adapter aux structurations urbaines régionales pour en tirer parti. Ainsi, des échanges croisés de migrants contraints lient Toulouse et Montpellier, Grenoble et Lyon, Dijon et Besançon, Metz et Nancy, Le Havre et Rouen, Tours et Orléans, Nantes et Rennes, ou encore Nantes et Angers.

La spécialisation de l'offre de formation universitaire contribue aussi à diversifier les chemins de l'émigration contrainte au départ d'un même centre. Un pôle universitaire peut se trouver dans la dépendance de plusieurs autres, soit que pour un même coût de déplacement, une même demande puisse être satisfaite dans plusieurs pôles, soit que la diversité des demandes émanant d'une même ville universitaire de départ se répartisse entre plusieurs centres en fonction des spécificités de l'offre de chacun. C'est le cas de Lyon: bien qu'au total excédentaire pour les migrations contraintes, elle n'en doit pas moins miser à la fois sur Marseille et sur Grenoble pour accueillir les émigrants qui ne trouvent pas sur place à satisfaire leur demande. A son tour, Grenoble alimente les flux d'entrée de Lyon et de Marseille, Chambéry ceux de Grenoble et de Lyon, et enfin Saint - Etienne ceux de Lyon, Grenoble et Clermont - Ferrand. Du fait d'une offre incomplète, Rennes alimente à la fois Paris, Nantes, Angers et Brest. Brest envoie des étudiants, contraints à émigrer, à Paris, à Rennes mais aussi à Nantes. De leur côté, les émigrants contraints d'Orléans se dirigent soit vers Paris, soit vers Tours, soit vers Bordeaux. 
Figures $n^{\circ} 3$ : Evaluation des migrations étudiantes contraintes

\section{a. Migrations contraintes entre villes de province}

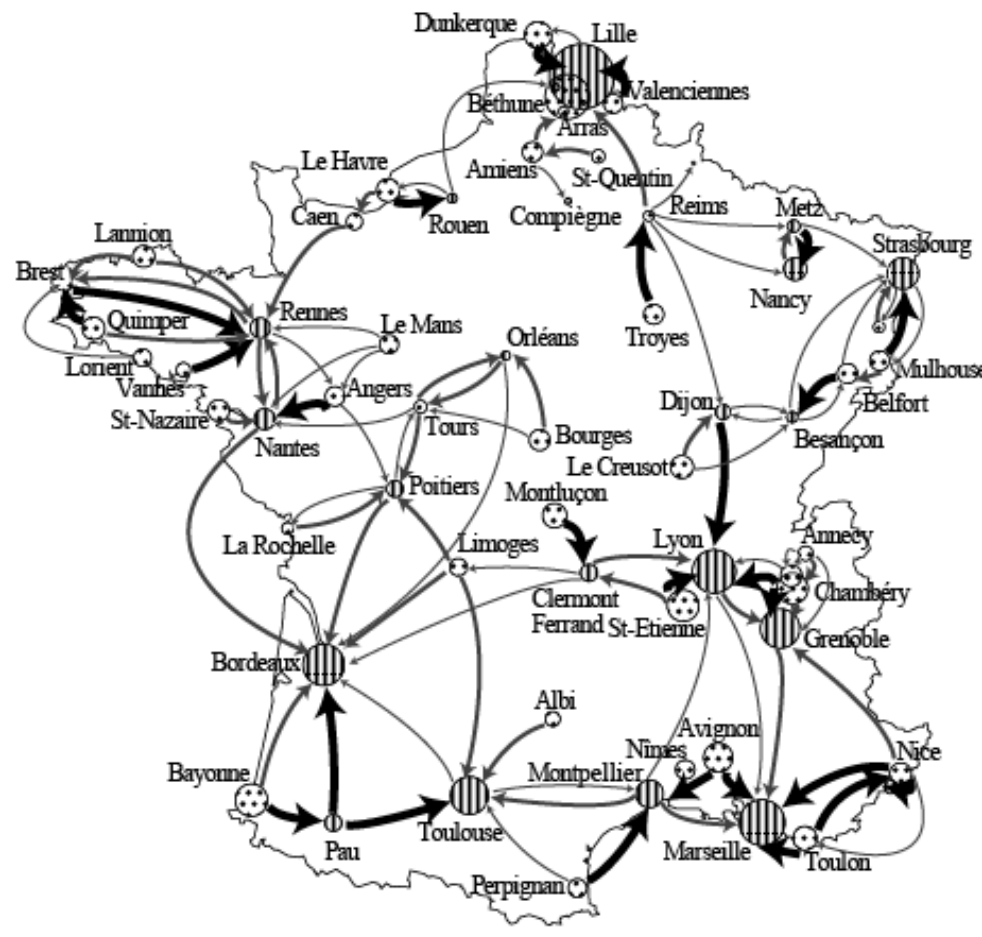

Principaux flux de migration contrainte* (nombre d'étudiants)

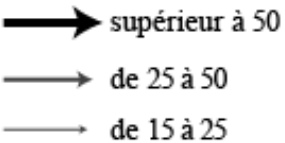

\section{b. Migrations contraintes vers Paris}

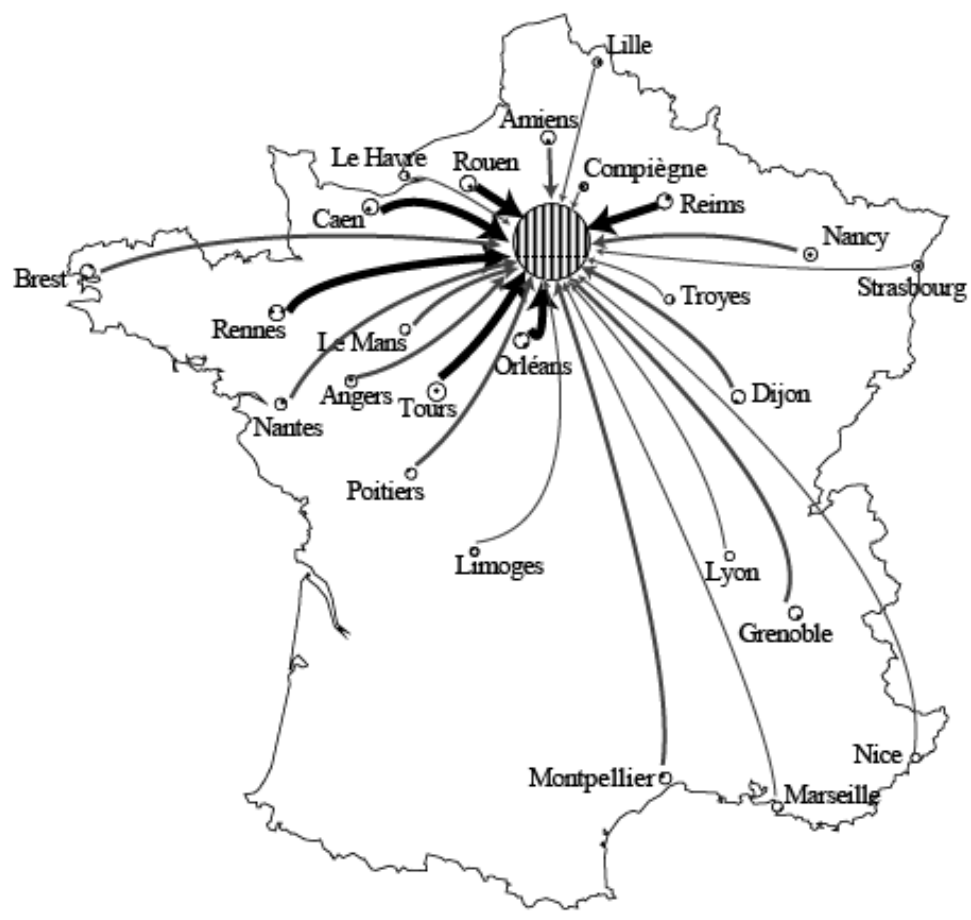

Solde des migrations contraintes* (nombre d'étudiants)

(III) supérieur à 300

(11) de 100 à 300

(2) de -100 à -300

$\odot$ inférieur à - 300

* Un étudiant est réputé avoir effectué une migration contrainte si la combinaison (cycle*discipline* formation) qui correspond à ces études en 1994 ne pouvait se trouver dans la ville où il a suivi ces études en 1993 (cf. Encadré $n^{\circ} 3$ ) 


\section{Plutôt que concurrences}

Si la mobilité géographique des étudiants est interprétée comme un facteur d'ouverture et d'enrichissement à la fois pour les migrants, qui élargissent leur univers intellectuel de référence, et pour les pôles universitaires qui voient ainsi se démultiplier les occasions de contact et, à terme, les probabilités de constructions communes diversifiées, les migrations contraintes peuvent être lues comme l'inscription de cette chance dans les structures. Les trop fortes dissymétries de ces échanges contraints en sont la dimension encore négative qui ne saurait être gommée avant longtemps si chaque ville poursuit implicitement la construction du même modèle universitaire polyvalent. Exigeant à la fois masse, diversité, et qualité dans la diversité, au point de placer la ville sur le plus grand nombre de segments possibles en situation relativement dominante dans le système, ce modèle n'arrive à maturité que sur le très long terme. En outre, cette lente montée en puissance doit compter avec une concurrence renforcée de l'ensemble des autres villes, dans un contexte de croissance des effectifs devenue en tendance relativement faible. Si elles maintiennent ce cap, certaines petites villes continueront de subir la migration contrainte sans pouvoir, en retour, en tirer avantage. A l'opposé, les grandes villes, mais aussi de plus petites, qui ont su et pu faire des choix originaux, jouent déjà d'interdépendances multiples et croisées pour conserver un potentiel migrations contraintes dont elles ont bien pris la mesure soit pour conforter leur dimension, soit pour renouveler leur population étudiante dans un jeu équilibré des échanges.

\section{CONCLUSION}

Les cadrages des migrations étudiantes entre villes universitaires ont rendu caduque ce qui peut être considéré comme un modèle de référence dans lequel le pôle universitaire parisien est censé exercer sur la province une ponction puissante et sélective, s'accentuant du premier au troisième cycle. Ce modèle ancien était basé sur l'intégration en particulier des migrations liées à l'entrée dans les Grandes Ecoles et les mouvements migratoires qui s'opéraient par anticipation en direction des Classes Préparatoires: il ne pouvait donc qu'accentuer le schématisme des représentations en vigueur.

Ces migrations étudiantes laissent finalement l'image d'un phénomène peu original du point de vue du jeu des facteurs liés à l'offre/demande des pôles universitaires et à la distance qui les sépare. Elles ressemblent fortement à celles que l'on observe pour l'ensemble de la population sur le territoire métropolitain. On peut alors se demander si la mobilité des étudiants entretient quelques rapports avec le changement de domicile des parents. Sur l'ensemble des étudiants qui changent de lieux d'étude entre 1993-94 et 1994-95, 95\% changent de département. Il est possible pour ces derniers de considérer que la migration correspond obligatoirement à un changement de ville universitaire. Or, seuls 3600 , soit $6 \%$ des étudiants migrants, semblent suivre leurs parents, en choisissant le même département de destination.

En outre, il convient d'être prudent dans l'interprétation des résultats déduits de l'analyse des migrations contraintes. Le fait que les pôles universitaires de petite taille aient un solde migratoire déficitaire par rapport aux grandes métropoles universitaires n'est pas en soit un signe d'échec mais la simple conséquence de propriétés structurelles liées à la taille des pôles universitaires et à la nature de l'offre de formation qu'ils fournissent. Le fait que les étudiants se déplacent vers des centres de formation situés à des échelons de plus en plus élevés de la hiérarchie urbaine au cours de l'avancement de leur formation n'a rien de pathologique et peut être vu, au contraire, comme un facteur positif dans la mesure où il génère des transitions 
progressives et évite une coupure trop brutale des attaches familiales des étudiants de premier et de deuxième cycles.

Ces mêmes migrations contraintes posent enfin le problème des pôles universitaires spécialisés dans une seule filière comme l'université de Compiègne. Ce type de pôle, en dépit du nombre de ses étudiants est capable d'être attractif à tous les passages de cycle. Il s'éloigne donc du type des centres universitaires dépendants, souvent généralistes et toujours incomplets. Le modèle de Compiègne demeure cependant atypique compte tenu de la concentration des soutiens privés et publics que son fonctionnement suppose. Sa reproductibilité est régulièrement évoquée, mais à ce jour jamais engagée. La limitation de nombreux pôles universitaires de petite taille à des formations généralistes de premier ou de deuxième cycle est la conséquence directe d'une règle beaucoup plus générale de fonctionnement des systèmes urbains liant la taille des villes et la rareté des services qu'elles sont en mesure de procurer en fonction de l'aire d'attraction qu'elles dominent. Au total, l'étude des migrations contraintes ne permet pas d'apporter des réponses tranchées au débat politique sur la poursuite ou l'abandon de la politique de déconcentration universitaire. Mais elle fournit un élément essentiel au débat en montrant que les politiques d'aménagement du territoire universitaire doivent nécessairement être intégrées à plusieurs échelons géographiques et s'appuyer sur les relations de concurrence et de complémentarités entre les pôles universitaires.

\section{BIBLIOGRAPHIE}

Auray J.-P. et alii (dir.), 1994, Encyclopédie d'Economie spatiale concepts - comportements organisations, Paris, Economica - ASRDLF, bibliothèque de Science régionale.

Baron M., Grasland C., Raulin E., Saint-Julien T., 1998, La mobilité géographique des étudiants des universités. Paris, convention de recherche CNRS-MENRT-DATAR.

Baron M., Grasland C., Saboulin (de) M., Saint-Julien T., 1999, Mobilité contrainte des étudiants des universités, déterminants individuels de la mobilité géographique. Paris, convention de recherche CNRS-MENRT.

Brocard M., Hérin R., Joly J. (coord.), 1996, Formation et recherche, Atlas de France, vol 4, Paris, RECLUS La Documentation Française.

Charlot (A), Chevalier (L) et Pottier (F), 1990, Qui forme pour qui ? Les régions, l'enseignement supérieur et l'emploi. Paris, Céreq.

DATAR (dir.), 1998, Développement Universitaire et développement territorial. L'impact du plan U2000 (1990-1995), Paris, La Documentation Française - DATAR.

De Gaudemar J.-P. (dir.), 1991, Formation et développement régional en Europe, Paris, La Documentation Française - DATAR.

Ertul S. (dir.), 2000 L'enseignement professionnel court post-baccalauréat (IUT-STS), Paris, Presses Universitaires de France, coll. Education et formation.

L'Espace Géographique, 1990-1991, « L'aménagement du territoire universitaire », vol. XIXXX, n 3, pp.193-213.

Felouzis G., 2001, « Les délocalisations universitaires et la démocratisation de l'enseignement supérieur », Revue Française de Pédagogie, n¹36, pp.53-63.

Fotheringham A.S. and O'Kelly M.E., 1989, Spatial interaction models : formulations and applications, Dordrecht, Kluwer Academic Publishers.

Frémont A., Hérin R. et Joly J., 1992, Atlas de la France universitaire. Paris, Reclus-La Documentation Française, Collection Dynamiques du territoire.

Musselin C., 2001, La longue marche des Universités françaises, Paris, PUF. 
Pihan J., 1998, «Les étudiants et leurs parents: cohabitation familiale et temps de déplacement », Espace, Populations, Sociétés, n², pp.181-198.

Pumain D., Saint-Julien T., 1989, Atlas des villes de France, Paris, RECLUS-La Documentation française, coll. Dynamiques des territoires.

Pumain D., Saint-Julien Th. (dir.), 1995, Atlas de France : L'espace des villes, Tome 12, Paris, RECLUS-La Documentation française.

Sen A., Smith TE., 1995, Gravity models of spatial interaction behaviour, Springer, Berlin.

\section{RESUME}

Le système universitaire français a connu de grands bouleversements au cours des années 90 . Des études nombreuses et à différents niveaux géographiques ont été menées pour caractériser la répartition des formations universitaires. En revanche, les travaux sont beaucoup moins nombreux et variés sur les migrations des étudiants au sein du système universitaire français. L'objet de cette note est de montrer les possibilités offertes par la mise à disposition des bases de données de la DEP et la manière dont l'étude de ces bases permet de cerner les migrations étudiantes. On apporte des éléments de réponse à quelques questions : que mesure-t-on ? Quels ordres de grandeur? Quelles relations particulières entre pôles universitaires ?

\section{SUMMARY}

French University System has changed a lot during the past decade. On one hand, numerous studies are made at different scales to understand how it is spread in French urban system. On the other hand, fewer are research works about how students migrate from one University City to an other. This work highlights part of the opportunities to know students' migrations from databases of Ministry of Education. We can partly answer to questions like: What is measured? How many people are concerned? What kind of relations exists between University cities? 